\title{
Parallel Fabrication of Nanogap Electrodes
}

\section{(Supplementary Information)}

Danvers E. Johnston ${ }^{1}$, Douglas R. Strachan ${ }^{1,2 *}$ and A. T. Charlie Johnson ${ }^{1,2 *}$

${ }^{1}$ Department of Physics and Astronomy, University of Pennsylvania, Philadelphia, Pennsylvania 19104, USA

${ }^{2}$ Department of Materials Science and Engineering, University of Pennsylvania, Philadelphia, Pennsylvania 19104, USA

*Email: (D. S.)drstrach@sas.upenn.edu, (C. J.) cjohnson@physics.upenn.edu. 


\section{Sample Fabrication}

Samples for FCE, consisting of a thin, narrow gold junction connected to thick gold leads, were fabricated using electron beam lithography and angle evaporation. Electron beam lithography was used to pattern two electrodes whose ends were separated by $150 \mathrm{~nm}$. For the array samples, junctions were spaced by $600 \mathrm{~nm}$. A bi-layer resist system was selected to guarantee a large undercut region, so that angle evaporation could be used to form the thin, narrow junction underneath a suspended resist bridge. ${ }^{1}$ The lower resist layer was $500 \mathrm{~nm}$ of a copolymer (MicroChem Corp, EL9), while the upper layer was $100 \mathrm{~nm}$ of polymethylmethacrylate (PMMA) (MicroChem, 950PMMAC2). After electron beam exposure, the pattern was developed in a 3:1 solution of isopropanol and methyl isobutyl ketone for 45 seconds. Thin film deposition was done in a thermal evaporator (base pressure below $10^{-6}$ Torr) equipped with a rotating sample stage. The metallization consisted of $3 \mathrm{~nm} \mathrm{Cr}$ deposited normal to the substrate, $12 \mathrm{~nm} \mathrm{Au}$ deposited with the sample rotated by $\pm 11^{\circ}$ to form the thin metal junction, and finally $30 \mathrm{~nm}$ Au deposited normal to the substrate.

\section{Circuit design considerations for parallel electromigration}

For $N$ junctions shorted together, as in the schematic of figure 1c from the main text, the stability condition is generalized to

$$
\frac{\partial P_{j}}{\partial R_{n j}}<\frac{\partial P_{i}}{\partial R_{n j}}
$$

where the resistance $R_{n j}$ of junction $j$ is increasing due to electromigration, and $P_{i}$ is the power dissipated at one of the other $(N-1)$ junctions. Analysis of equation (1) gives

$$
\frac{2 R_{L} R_{\|}}{R_{L}+R_{\|}} \cdot\left[\frac{R_{n j}}{\left(R_{C}+R_{n j}\right)^{2}}-\frac{R_{n i}}{\left(R_{C}+R_{n i}\right)^{2}}\right]+\frac{\left(R_{C}-R_{n j}\right)}{\left(R_{C}+R_{n j}\right)}<0,
$$

where $R_{\|} \equiv 1 / \sum_{i=1}^{N}\left(R_{C}+R_{n i}\right)^{-1}$. The junction resistances $R_{n i}$ and $R_{n j}$ are all roughly equal so the first 
term in equation (2) is zero to lowest order. The remaining term in equation (2) requires that $R_{C}<R_{n i} \approx R_{n j}$, which is the resistive condition for stable parallel electromigration. As $R_{n j}$ increases due to electromigration, the left side of equation (2) remains negative which maintains the necessary inequality.

\section{Sequential gap formation and melting}

Melting is discernible by scanning electron microscopy (SEM) in some gaps formed through the sequential electromigration process with a spherical build-up of $\mathrm{Au}$ (supplementary figure 1).

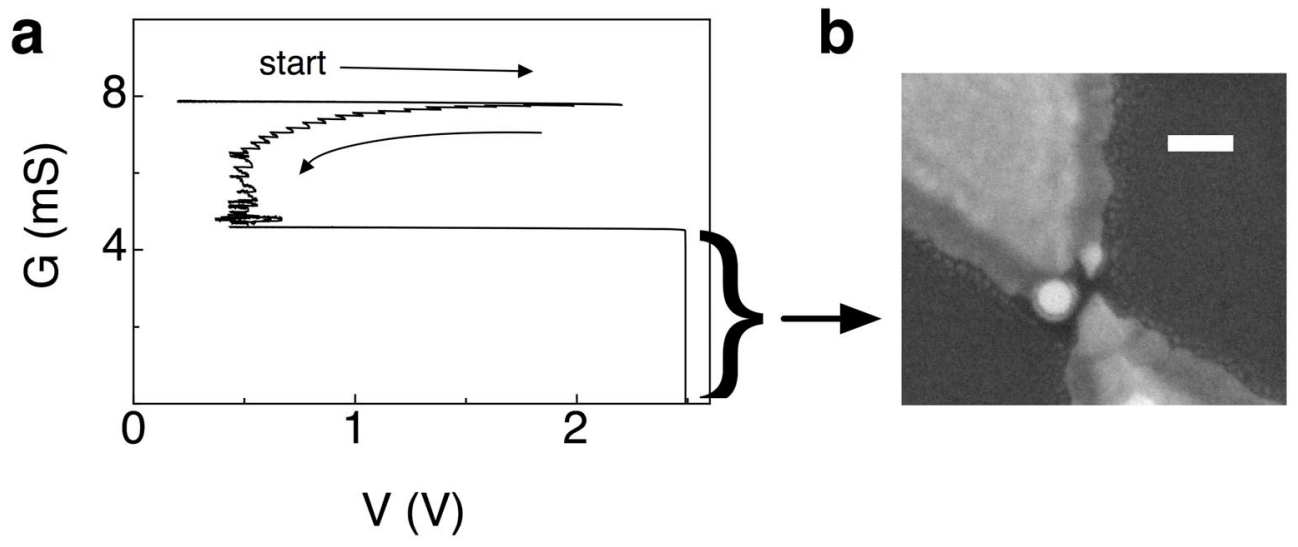

Supplementary figure 1. Melting due to sequential electromigration. (a) The sequential gap formation discussed in the main text. (b) SEM image of the second gap formed showing a spherical build-up of Au. (Scale bar is $100 \mathrm{~nm}$.)

\section{Single-ramp electromigration of 16-junction array}

In striking contrast to feedback controlled electromigration (FCE), the majority of junctions in an array of electrically shorted $\mathrm{Au}$ junctions with low inter-junction resistances are catastrophically destroyed in a single-ramp process. The resulting gaps exhibit a range of gaps sizes with the majority (9 out of 16) having widths larger than $0.5 \mu \mathrm{m}$ (supplementary figure 2). 

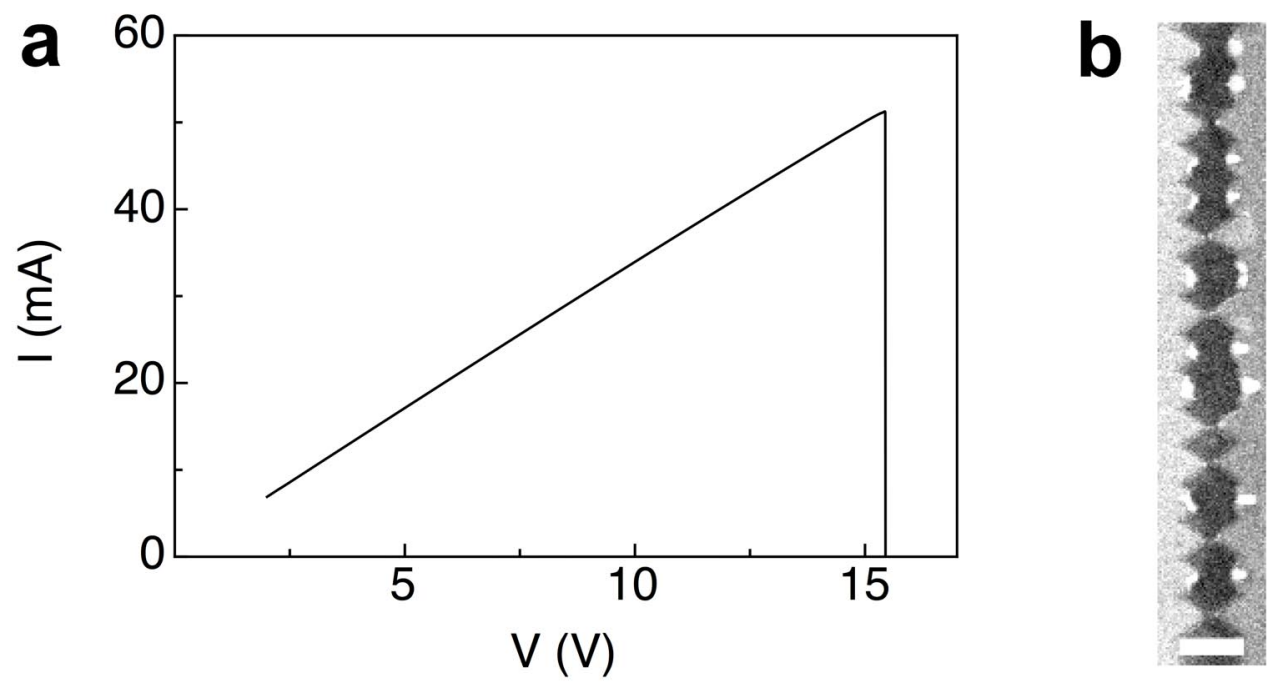

Supplementary figure 2. Single voltage ramp electromigration of 16 Au junction array. (a) An applied voltage is ramped across the array at $58 \mathrm{mV} / \mathrm{sec}$ until failure occurs. (b) SEM image after failure. (Scale bar is $1 \mu \mathrm{m}$.)

\section{REFERENCES}

1. Dolan, G. J. Appl. Phys. Lett. 1977, 31, 337. 